\title{
Progenitors of binary black hole mergers detected by LIGO
}

\author{
Konstantin Postnov and Alexander Kuranov \\ Sternberg Astronomical Institute, Moscow M.V.Lomonosov State University \\ 13, Universitetskij pr., 119234 Moscow, Russia \\ email: pk@sai.msu.ru
}

\begin{abstract}
Possible formation mechanisms of massive close binary black holes that can merge in the Hubble time to produce powerful gravitational wave bursts detected during advanced LIGO O1 science run are briefly discussed. The pathways include the evolution from field lowmetallicity massive binaries, the dynamical formation in globular clusters and primordial black holes. Low effective black hole spins inferred for LIGO GW150914 and LTV151012 events are discussed. Population synthesis calculations of the expected spin and chirp mass distributions from the standard field massive binary formation channel are presented for different metallicities (from zero-metal Population III stars up to solar metal abundance). We conclude that that merging binary black holes can contain systems from different formation channels, discrimination between which can be made with increasing statistics of mass and spin measurements from ongoing and future gravitational wave observations.
\end{abstract}

Keywords. black hole physics, stars: evolution, (stars:) binaries (including multiple): close

\section{Introduction and historical remarks}

The epochal discovery of the first gravitational wave source GW150914 from coalescing binary black hole (BH) system (Abbott et al. 2016d) not only heralded the beginning of gravitational wave astronomy era, but also stimulated a wealth of works on fundamental physical and astrophysical aspects of the formation and evolution of binary BHs. The LIGO detection of GW150914 and of the second robust binary BH merging event GW151226 (Abbott et al. 2016c) enables BH masses and spins before the merging, the luminosity distance to the sources and the binary $\mathrm{BH}$ merging rate in the Universe to be estimated (Abbott et al. 2016b). Astrophysical implications of these measurements were discussed, e.g., in Abbott et al. (2016e,a).

This discovery of gravitational waves from coalescing binary BHs was long awaited. Evolution of massive binary systems was elaborated in the 1970s to explain a rich variety of newly discovered galactic X-ray binaries (van den Heuvel \& Heise 1972; Tutukov et al. 1973). Formation of two relativistic compact remnants (neutron stars (NSs) or black holes) naturally followed from the binary evolution scenario (Tutukov \& Yungelson 1973; Flannery \& van den Heuvel 1975). At the dawn of the LIGO Project, Tutukov and Yungelson (Tutukov \& Yungelson 1993) calculated, using the standard assumptions of massive binary evolution, the expected galactic merging rate of binary NSs and BHs. They pointed out that although the galactic merging rate of binary NSs is much larger than that of binary $\mathrm{BHs}$, their detection rates by gravitational-wave interferometers can be comparable due to the strong dependence of the characteristic GW amplitude $h_{c}$ on the total mass $M=M_{1}+M_{2}$ of the coalescing binaries, $h_{c} \sim M^{5 / 2}$. A few years later, independent population synthesis calculations by the Scenario Machine code were reported in a series of papers (Lipunov et al. 1997b,c,a). They showed that in a wide range of possible $\mathrm{BH}$ formation parameters (masses,kick velocities) and under standard 
assumptions of the massive star evolution, the detection rate of binary $\mathrm{BH}$ mergings should be much higher than that of binary NSs, and the first LIGO event should most likely to be a binary $\mathrm{BH}$ merging. Interestingly, the mean $\mathrm{BH}$ masses known at that time from dynamical measurements in galactic BH X-ray binaries were about $10 M_{\odot}$, which forced (cautiously) the authors of (Lipunov et al. 1997a) to fix the parameter $k_{B H}=M_{B H} / M_{c}$, where $M_{c}$ is the mass of the star before the collapse, around $\sim 0.3$ (see Fig. 4 in that paper) in order to produce the chirp mass of coalescing binary BHs around $15 M_{\odot}$. Taking $k_{B H}=1$, one immediately obtains the $\mathrm{BH}$ masses around 30-40 $M_{\odot}$, which seemed outrageously high at that time.

Starting from the end of the 1990s, various groups have used different population synthesis codes to calculate the merging rates of double compact objects (see especially many papers by the Polish group based on the StarTrack code (Belczynski et al. 2002; Dominik et al. 2012)), yielding a wide range of possible BH-BH merging rates (see e.g. Table 6 in Postnov \& Yungelson (2014)). Clearly, the degeneracy of binary evolution and $\mathrm{BH}$ formation parameters has been so high (Abadie et al. 2010) that only real observations could narrow the wide parameter range.

\section{Standard scenario of binary BH formation}

The standard scenario of double BH formation from field stars is based on wellrecognized evolution of single massive stars Woosley et al. (2002). To produce a massive $\mathrm{BH}$ with $M \simeq 10 M_{\odot}$ in the end of evolution, the progenitor star should have a large mass and low mass-loss rate. The mass-loss rate is strongly dependent on the metallicity, which plays the key role in determining the final mass of stellar remnant (see Spera et al. (2015) and N. Yusof's contribution in this conference). The metallicity effects were included in the population synthesis calculations (Dominik et al. 2013), and the most massive BHs were found to be produced by the low-metallicity progenitors. Here early metal-free Population III stars provide an extreme example, see calculations by Kinugawa et al. (2014); Hartwig et al. (2016). After the discovery of GW150914, several independent population synthesis calculations were performed to explain the observed masses of binary $\mathrm{BH}$ in GW150914 and the inferred binary BH merging rate $\sim 9-240 \mathrm{Gpc}^{-3} \mathrm{yr}^{-1}$ (Abbott et al. 2016b) (see, among others, e.g. Belczynski et al. 2016; Eldridge \& Stanway 2016; Lipunov et al. 2017).

In addition to the metallicity that affects the intrinsic evolution of the binary components, the most important uncertainty in the binary evolution is the efficiency of the common envelope (CE) stage which is required to form a compact double BH binary merging in the Hubble time. The common envelope stage remains a highly debatable issue. For example, in recent hydro simulations (Ohlmann et al. 2016) a low CE efficiency was found, while successful CE calculations were reported by other groups (see, e.g., N. Ivanova contribution at this conference). Another recent study (Pavlovskii et al. 2017) argues that it is possible to reconcile the $\mathrm{BH}$ formation rate through the $\mathrm{CE}$ channel taking into account the stability of mass transfer in massive binaries in the Hertzsprung gap stage, which drastically reduces the otherwise predicted overproduction of binary $\mathrm{BH}$ merging rate in some population synthesis calculations. Also, the so-called stable 'isotropic re-emission' mass transfer mode can be realized in high-mass X-ray binaries with massive BHs, thus helping to avoid the merging of the binary system components in the common envelope (van den Heuvel et al. 2017). This stable mass transfer mode can explain the surprising stability of kinematic characteristics observed in the galactic microquasar SS433 (Davydov et al. 2008). 
Of course, much more empirical constraints on and hydro simulations of the common evolution formation and properties are required, but the formation channel with common envelope of binary BHs with properties similar to GW150914 remains quite plausible.

\section{Other scenarios}

To avoid the ill-understood common envelope stage, several alternative scenarios of binary BH formation from massive stars were proposed. For example, in short-period massive binary systems chemically homogeneous evolution due to rotational mixing can be realized. The stars remain compact until the core collapse, and close binary BH system is formed without common envelope stage (Mandel \& de Mink 2016; de Mink \& Mandel 2016; Marchant et al. 2016). In this scenario, a pair of nearly equal massive BHs can be formed with the merging rate comparable to the empirically inferred from the first LIGO observations.

Another possible way to form massive binary BH system is through dynamical interactions in a dense stellar systems (globular clusters). This scenario was earlier considered by Sigurdsson \& Hernquist (1993). In the core of a dense globular clusters, stellar-mass BH form multiple systems, and $\mathrm{BH}$ binaries are dynamically ejected from the cluster. This mechanism was shown to be quite efficient in producing $30+30 M_{\odot}$ merging binary BHs (Rodriguez et al. 2016b), and binary BH formed in this way can provide a substantial fraction of all binary BH mergings in the local Universe (Rodriguez et al. 2016a).

Finally, there can be more exotic channels of binary $\mathrm{BH}$ formation. For example, primordial black holes ( $\mathrm{PBHs}$ ) formed in the early Universe can form pairs which could be efficient sources of gravitational waves (Nakamura et al. 1997). After the discovery of GW150914, the interest to binary PBHs has renewed (Bird et al. 2016). Stellar-mass PBHs can form a substantial part of dark matter in the Universe (Carr et al. 2016). The PBHs formed at the radiation-dominated stage can form pairs like GW150914 with the merging rate compatible with empirical LIGO results, being only a small fraction of all dark matter (Eroshenko 2016; Sasaki et al. 2016). Different class of PBHs with a universal log-normal mass spectrum produced in the frame of a modified Affleck-Dine supersymmetric baryogenesis (Dolgov \& Silk 1993; Dolgov et al. 2009) were shown to be able to match the observed properties of GW150914 Blinnikov et al. (2016).

\section{Low spins of BH in GW150914 and LTV151012 events}

In the framework of general relativity, a $\mathrm{BH}$ is fully characterized by its mass $M$ and dimensionless angular momentum $a=J / M$ (in geometrical units $G=c=1$ ) (the possible $\mathrm{BH}$ electric charge is negligible in real astrophysical conditions). The LIGO observations enable measurements of both masses of the coalescing $\mathrm{BH}$ components, $M_{1}$ and $M_{2}$, and the chirp mass that determines the strength of gravitational wave signal $\mathcal{M}=\left(M_{1} M_{2}\right)^{3 / 5} / M^{1 / 5}$. From the analysis of waveforms at the inspiral stage, individual $\mathrm{BH}$ spins before the merging are poorly constrained, but their mass-weighted total angular momentum parallel to the orbital angular momentum, $\chi_{e f f}$, can be estimated with good accuracy (Abbott et al. 2016b) $\dagger$. The O1 LIGO detections suggest that the most massive GW150914 and (less certain) LTV151012 have very low $\chi_{\text {eff }} \simeq 0$.

This observational fact has important evolutionary implications (see Kushnir et al. (2016); Hotokezaka \& Piran (2017)). It suggests a very slow rotation of BH progenitors,

$\dagger$ The parameter $\chi_{e f f}=\left(M_{1} \chi_{1}+M_{2} \chi_{2}\right) / M$, where $\chi_{i}=a_{i} \cos \theta_{i}$ with $\theta_{i}$ being the angle between the angular momentum of the $\mathrm{i}$-th $\mathrm{BH}$ and orbital angular momentum of the binary system. 


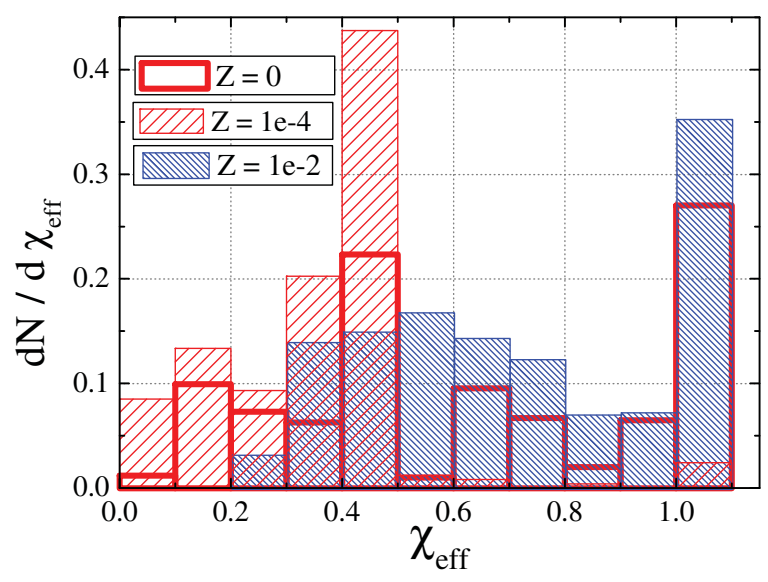

Figure 1. Distribution of the effective binary BH spin parameter $\chi_{e f f}$ before the merging for different stellar metallicities.

which by itself strongly constraints, for example, chemically homogeneous pathways mentioned above in which tidally induced rotation of the close binary components plays the key role. Massive stars are observed to be rapid rotators. No significant angular momentum loss is expected during their evolution with low mass loss rate by stellar wind and at the pre-collapse stage as required to produce massive BHs (Spera et al. 2015). Note that low effective spin values can imply either small intrinsic BH spins $a \sim 0$, or unusual orientations of $\mathrm{BH}$ spins with respect to the orbital angular momentum at the inspiral stage. The last case can well be reconciled with the dynamical formation scenario (Rodriguez et al. 2016a), where the BH spins are not expected to be correlated with the orbital angular momentum. In the $\mathrm{PBH}$ scenario, $\mathrm{BH}$ spins must be zero as there are no vorticity in primordial cosmological perturbations.

Therefore, the mass-spin distribution of BHs can serve as a sensitive tool to discriminate between different astrophysical formation channels of coalescing massive binary $\mathrm{BHs}$. To estimate the spin distribution of $\mathrm{BH}$ remnants in binaries, it is necessary to know how to treat the spin evolution of the stellar core, which is ill-understood and strongly model-dependent. One possible approach is to match theoretical predictions of the core rotation with observed period distribution of the young neutron stars observed as radio pulsars (Postnov et al. 2016). Initially, a star is assumed to rotate rigidly, but after the main sequence the star can be separated in two parts - the core and the envelope, with some effective coupling between these two parts. The coupling between the core and envelope rotation can be mediated by magnetic forces, internal gravity waves (see Fuller et al. (2015) and J.Fuller's talk at this conference), etc. The validity of such an approach was checked by direct MESA calculations of the rotational evolution of a $15 M_{\odot}$ star (Postnov et al. 2016). It was found that the observed period distribution of young pulsars can be reproduced if the effective coupling time between the core and envelope is $\tau_{c}=5 \times 10^{5}$ years (see Fig. 1 in Postnov et al. (2016)). Below we shall assume that this time is also applicable to the evolution of very massive stars leaving behind $\mathrm{BH}$ remnants.

Each angular momentum of the main-sequence components of the initial binary is assumed to be arbitrarily distributed in space, its absolute value being connected to the initial stellar mass using the empirical relation between the equatorial rotation velocity of a star with its mass $v_{r o t}=330 M_{0}^{3.3} /\left(15+M_{0}^{3.45}\right) \mathrm{km} \mathrm{s}^{-1}$ (here $M_{0}$ is in solar units). It was assumed that the rotation of the stellar envelope gets synchronized with the 

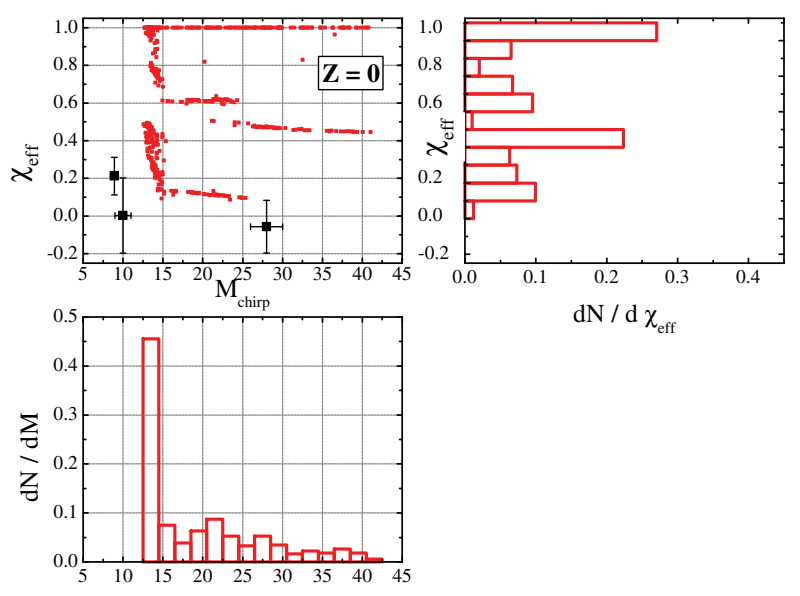

$\mathrm{dN} / \mathrm{d} \chi_{\text {eff }}$
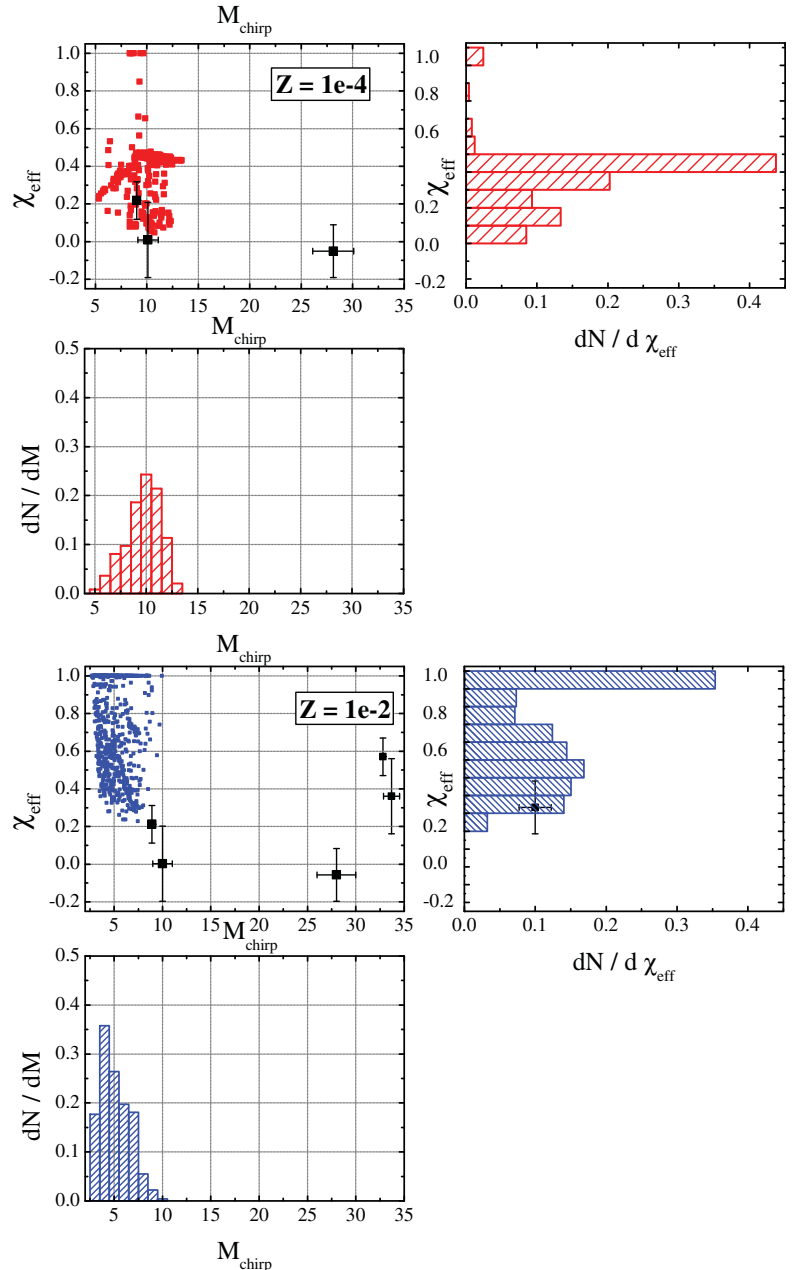

$\mathrm{dN} / \mathrm{d} \chi_{\mathrm{eff}}$

Figure 2. $\mathcal{M}-\chi_{\text {eff }}$ plane for different stellar metallicities. Filled squares show the observed BH-BH systems (Abbott et al. 2016b), GW150914, LTV151012 and GW151226, in order of decreasing chirp mass. 

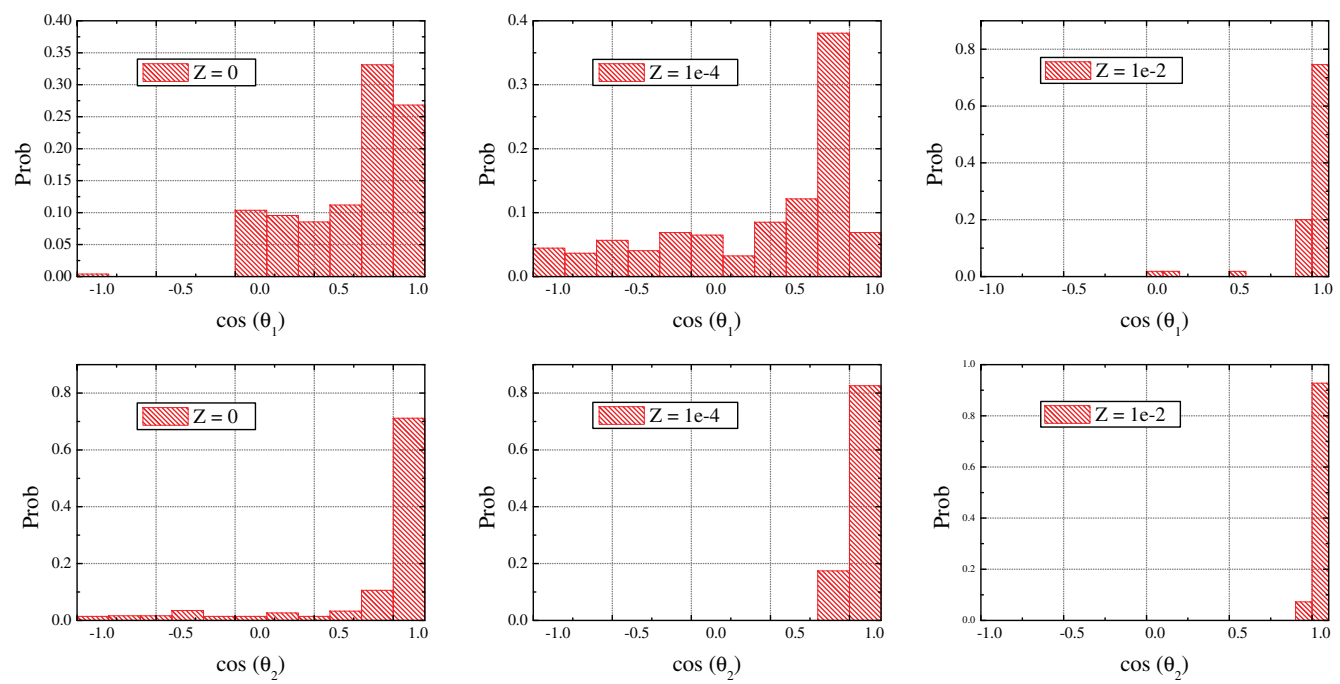

Figure 3. BH spin misalignments with orbital angular momentum (in terms of $\cos \theta$ ) in coalescing binary BHs for different stellar metallicities. Top and bottom row correspond to $M_{1}$ and $M_{2}$, respectively.

orbital motion with the characteristic synchronization time $t_{\text {sync }}$, and the process of tidal synchronization was treated as in the BSE code (Hurley et al. 2002). Due to intrinsic misalignment of the spin vectors of the stars with the binary orbital angular momentum $\hat{L}$, we separately treated the core-envelope coupling for the spin components parallel and perpendicular to $\hat{L}$. On evolutionary stages prior to the compact remnant formation, for each binary component we assumed that due to tidal interactions the parallel component of the stellar envelope spin $J_{\|}$gets synchronized with the orbital motion on the characteristic time $t_{\text {sync }}$, while the normal spin component $J_{\perp}$ of the stellar envelope decreases due to the tidal interaction in the binary system the on the same characteristic time scale, which leads to the secular evolution of the spin-orbit misalignment. The parallel component of the envelope spin also evolves due to the core-envelope interaction with the characteristic time $\tau_{c}$. These processes were added to the updated BSE population synthesis code.

With these additions, the population synthesis of typically 100000 binaries per run has been carried out for different parameters of binary evolution (the common envelope stage efficiency $\alpha_{C E}$, stellar metallicities etc.). No generic BH kick was assumed. The results of calculations of $\mathrm{BH}$ spin distributions for different stellar metallicites and for the standard CE efficiency parameter $\alpha_{C E}=1$ are shown in Fig. 1. Evolution of zero-metallicity (primordial Population III) stars was parametrized as in Kinugawa et al. (2014). Fig. 2 shows the plot of coalescing binary BHs on the $\mathcal{M}-\chi_{\text {eff }}$ plane for different metallicities. $\mathrm{BH}$ spin misalignments with orbital angular momentum in coalescing binary BHs for different stellar metallicities are presented in Fig. 3.

A detailed analysis of these simulations will be published elsewhere (Postnov \& Kuranov, in preparation), but the main conclusions can be drawn from Figs. 1-3. It is seen (expectedly) from Fig. 1 that the effective spin $\chi_{\text {eff }}$ of binary $\mathrm{BH}$ from field massive stars (the standard formation scenario) is distributed in a wide range, but the $\mathcal{M}-\chi_{\text {eff }}$ plot (Fig. 2) suggests that large chirp masses can hardly have $\chi_{e f f} \simeq 0$. This (model) 
result can signal potential difficulty in explaining the most massive merging $\mathrm{BH}$ binaries by this formation channel only. Fig. 3 suggests that even in the absence of BH kicks, which were assumed in the present calculations, the BH spin misalignments can be quite high even for field binaries.

\section{Conclusions}

Presently, there are different astrophysical pathways of producing massive binary BHs that merge in the Hubble time. They can be formed from low-metallicity massive field stars, primordial Pop III remnants, can be results of dynamical evolution in dense stellar clusters or even primordial black holes. It is not excluded that all channels contribute to the observed binary BH population. For example, the discovery of very massive Schwarzschild BHs would be difficult to reconcile with the standard massive binary evolution, but can be naturally explained in the PBH scenario (Blinnikov et al. 2016).

As of the time of writing, another six event candidates were reported by the LIGO collaboration from the analysis of 67 days of joint operation of two LIGO interferometers during O2 run (see http://ligo.org/news/index.php\#02Apr2017update). With the current LIGO sensitivity, the detection horizon of binary $\mathrm{BH}$ with masses around 30 $M_{\odot}$ reaches $700 \mathrm{Mpc}$. So far the statistics of binary $\mathrm{BH}$ merging rate as a function of $\mathrm{BH}$ mass as inferred from three reported LIGO O1 events is consistent with a power-law dependence, $d R / d M \sim M^{-2.5}$ (Hotokezaka \& Piran 2017), which does not contradict the general power-law behavior of the stellar mass function. Clearly, more statistics of $\mathrm{BH}$ masses and spins inferred from binary $\mathrm{BH}$ mergings is required to distinguish between the possible binary $\mathrm{BH}$ populations which can exist in the Universe.

Acknowledgements. KP acknowledges the support from RSF grant 16-12-10519.

\section{References}

Abadie, J. et al. 2010, Classical and Quantum Gravity, 27, 173001

Abbott, B.P. et al. 2016a, ApJL, 818, L22

Abbott, B.P. et al. 2016b, Physical Review X, 6, 041015

Abbott, B.P. et al. 2016c, Physical Review Letters, 116, 241103

Abbott, B.P. et al. 2016d, Physical Review Letters, 116, 061102

Abbott, B.P. et al. 2016e, ApJL, 833, L1

Belczynski, K., Holz, D. E., Bulik, T., \& O'Shaughnessy, R. 2016, Nature, 534, 512

Belczynski, K., Kalogera, V., \& Bulik, T. 2002, ApJ, 572, 407

Bird, S. et al. 2016, Physical Review Letters, 116, 201301

Blinnikov, S., Dolgov, A., Porayko, N. K., \& Postnov, K. 2016, JCAP, 11, 036

Carr, B., Kühnel, F., \& Sandstad, M. 2016, Phys. Rev. D, 94, 083504

Davydov, V. V., Esipov, V. F., \& Cherepashchuk, A. M. 2008, Astronomy Reports, 52, 487

de Mink, S. E. \& Mandel, I. 2016, MNRAS, 460, 3545

Dolgov, A. \& Silk, J. 1993, Phys. Rev. D, 47, 4244

Dolgov, A. D., Kawasaki, M., \& Kevlishvili, N. 2009, Nuclear Physics B, 807, 229

Dominik, M. et al. 2012, ApJ, 759, 52

Dominik, M. et al. 2013, ApJ, 779, 72

Eldridge, J. J. \& Stanway, E. R. 2016, MNRAS, 462, 3302

Eroshenko, Y. N. 2016, ArXiv e-prints

Flannery, B. P. \& van den Heuvel, E. P. J. 1975, A\&A, 39, 61

Fuller, J., Cantiello, M., Lecoanet, D., \& Quataert, E. 2015, ApJ, 810, 101

Hartwig, T. et al. 2016, MNRAS, 460, L74

Hotokezaka, K. \& Piran, T. 2017, ArXiv e-prints 
Hurley, J. R., Tout, C. A., \& Pols, O. R. 2002, MNRAS, 329, 897

Kinugawa, T., Inayoshi, K., Hotokezaka, K., Nakauchi, D., \& Nakamura, T. 2014, MNRAS, 442, 2963

Kushnir, D., Zaldarriaga, M., Kollmeier, J. A., \& Waldman, R. 2016, MNRAS, 462, 844

Lipunov, V.M., Postnov, K.A., \& Prokhorov, M.E. 1997a, Astronomy Letters, 23, 492

Lipunov, V.M., Postnov, K.A., \& Prokhorov, M.E. 1997b, New Astron., 2, 43

Lipunov, V.M., Postnov, K.A., \& Prokhorov, M.E. 1997c, MNRAS, 288, 245

Lipunov, V. M. et al. 2017, New Astron., 51, 122

Mandel, I. \& de Mink, S. E. 2016, MNRAS, 458, 2634

Marchant, P., Langer, N., Podsiadlowski, P., Tauris, T. M., \& Moriya, T. J. 2016, A\& A, 588, A50

Nakamura, T., Sasaki, M., Tanaka, T., \& Thorne, K. S. 1997, ApJL, 487, L139

Ohlmann, S. T., Röpke, F. K., Pakmor, R., \& Springel, V. 2016, ApJL, 816, L9

Pavlovskii, K., Ivanova, N., Belczynski, K., \& Van, K. X. 2017, MNRAS, 465, 2092

Postnov, K. A., Kuranov, A. G., Kolesnikov, D. A., Popov, S. B., \& Porayko, N. K. 2016, MNRAS, 463, 1642

Postnov, K. A. \& Yungelson, L. R. 2014, Living Reviews in Relativity, 17, 3

Rodriguez, C.L., Chatterjee, S., \& Rasio, F.A. 2016a, Phys. Rev. D, 93, 084029

Rodriguez, C.L., Haster, C.J., Chatterjee, S., Kalogera, V., \& Rasio, F.A. 2016b, ApJL, 824, L8

Sasaki, M., Suyama, T., Tanaka, T., \& Yokoyama, S. 2016, Physical Review Letters, 117, 061101

Sigurdsson, S. \& Hernquist, L. 1993, Nature , 364, 423

Spera, M., Mapelli, M., \& Bressan, A. 2015, MNRAS, 451, 4086

The LIGO Scientific Collaboration et al. 2016, ArXiv e-prints

Tutukov, A. \& Yungelson, L. 1973, Nauchnye Informatsii, 27, 70

Tutukov, A., Yungelson, L., \& Klayman, A. 1973, Nauchnye Informatsii, 27, 3

Tutukov, A. V. \& Yungelson, L. R. 1993, MNRAS, 260, 675

van den Heuvel, E. P. J. \& Heise, J. 1972, Nature Physical Science, 239, 67

van den Heuvel, E. P. J., Portegies Zwart, S. F., \& de Mink, S. E. 2017, ArXiv e-prints

Woosley, S. E., Heger, A., \& Weaver, T. A. 2002, Reviews of Modern Physics, 74, 1015 